\title{
ON GOLDIE THEOREMS FOR QUOTIENT RINGS
}

\author{
GEORGE SZETO
}

1. Introduction. It is well known that a ring has a quotient ring if and only if it satisfies the Ore condition. The structure of the quotient ring of a ring with the Ore condition has been intensively investigated. In particular, A. W. Goldie ([4], [5], and [6]) proved that a ring is prime Goldie if and only if its quotient ring is Artinian simple, and that a ring is semiprime Goldie if and only if its quotient ring is Artinian semisimple. The purpose of the present paper is to generalize the above theorems of Goldie to a ring with an A-biregular or weakly A-biregular quotient ring, where an $A$-biregular ring $R$ is a ring whose stalks of the Pierce sheaf [9] induced by $R$ are Artinian simple rings, and a weakly $A$-biregular ring is a ring whose stalks of the Pierce sheaf induced by $R$ are Artinian semisimple. Our theorem is the following: Let $R$ be a ring with the identity 1 in which every non-zero-divisor of the stalks of the Pierce sheaf is lifted to a non-zero-divisor of $R$. If the stalks are prime Goldie (semiprime Goldie), then the quotient ring of $R$ exists and is A-biregular (weakly A-biregular). A counterexample will be given to show that the converse does not always hold. However, when no extra central idempotents are added to the quotient ring from those of $R$, the theorem is reversible. Moreover, as pointed out by G. Bergman [1], the set of idempotents of the quotient ring of a commutative ring may be larger than the set of those of the ring. We shall examine this fact for a non-commutative ring in detail, and our results characterize a weakly A-regular quotient ring being A-biregular, equivalently, semiprime Goldie stalks being prime Goldie. Since prime Goldie rings and semiprime Goldie rings admit only a finite number of central idempotents, our study is a generalization of Goldie theorems for quotient rings to a class of rings with infinite central idempotents.

The author wishes to thank the referee for his valuable suggestions and comments and for bringing [2] to our attention.

2. Preliminaries. The ring $R$ is called a (left) Goldie ring if $R$ satisfies the ACC condition on (left) annihilators, and if $R$ has no infinite direct sum of (left) ideals ([7], p. 62). The ring $R$ with the left Ore condition is a ring such that for any $a, s$ in $R$ with $s$ a non-zero-divisor, there exist $b, t$ in $R$ with $t$ a non-zero-divisor such that $t a=b s$. It is well known that $R$ satisfies the (left) Ore condition if and only if it has a

Received by the editors on April 4, 1977 and in revised form on October 12, 1977. 
(left) quotient ring ([7], p. 61). For the definitions of prime, semiprime rings see [7].

Let $R$ be a ring with the identity $1, B(R)$ the set of central idempotents of $R,(B(R), \vee, \wedge)$ the usual Boolean algebra, and Spec $B(R)$ the Boolean spectrum of the set of maximal ideals of $(B(R), \vee, \wedge)$. We note that $\operatorname{Spec} B(R)$ has an open base, $\Gamma(e)=\{x$ in Spec $B(R) \mid e$ is not in $x$ \} for $e$ in $B(R)$. R. Pierce [9] proved that a sheaf (the Pierce sheaf) of rings $R / x R$ for $x$ in Spec $B(R)$ is defined, and that $R$ is isomorphic with the ring of sections of the sheaf (that is, the ring of continuous functions from Spec $B(R)$ to the sheaf). A number of algebraic properties and applications to the sheaf representation theory have been found by R. Pierce [9], O. Villamayor and D. Zelinsky [10], G. Bergman [1], A. Magid [8], F. DeMeyer [3], and others. We shall employ some basic properties as given in [9] and [10].

Throughout, we assume that $R$ is a ring with 1 , and that all quotient rings and the Ore condition are left sided. Denote the quotient ring of the ring $R$ with the Ore condition by $Q(R)$.

3. Quotient rings and Goldie theorems on quotient rings. R. Pierce [9] and G. Bergman [1] studied a class of rings $R$ in which the support $\operatorname{Sup}(r)$ of any element $r$ in $R$ is both open and closed in Spec $B(R)$. Now we are interested in a class of rings $R$ in which the subset $T(r)=\left\{x\right.$ in Spec $B(R) \mid r_{x}$, the image of $r$ in $R / x R$, is a non-zero-divisor in the stalk $R / x R$, for each $r$ in $R$, is both open and closed. When $R$ is a p.p. ring [1] such that the left annihilator of each element in $R$ is the annihilator of some $e$ in $B(R), T(r)$ is both open and closed. By using the usual sheaf technique, it is not difficult to show:

Proposition 3.1. If $T(r)$ is both open and closed for each $r$ in $R$, then $R$ satisfies the Ore condition if and only if so does each stalk $R / x R$.

Proposition 3.2. If $T(r)$ is both open and closed for each $r$ in $R$, then $S$ is the set of non-zero-divisors of $R$ if and only if $(S)_{x}$, the image of $S$ in $R / x R$, is the set of non-zero-divisors of $R / x R$ for each $x$ in $\operatorname{Spec} B(R)$.

In this section, we show that, for the ring $R$ as given in Proposition 3.1, the quotient ring $Q(R)$ of $R$ is isomorphic with the ring of sections of the sheaf of $Q(R / x R)$ over Spec $B(R)$. Then, we generalize the Goldie theorems on quotient rings to rings with infinite central idempotents. Throughout the section, $T(r)$ is assumed both open and closed for each $r$ in $R$. Let $S$ be the set of non-zero-divisors of $R$. We note that $S_{x}$ is the set of non-zero-divisors of $R / x R$.

Lemma 3.3. If $R / x R$ satisfies the Ore condition, $Q(R / x R)$ is isomorphic with $Q(R) / x Q(R)$ for each $x$ in $\operatorname{Spec} B(R)$. 
Proof. By hypothesis, $S_{x}$ is the set of non-zero-divisors of $R / x R$, so the map: $\left(s^{-1} r\right)_{x} \rightarrow\left(s^{-1}\right)_{x} r_{x}$ is an isomorphism, where $s$ is in $S$ and $r$ in $R$.

Let $R$ be a ring with the Ore condition. Similar to Lemma 3.1 (ii implies iii) in [1], we have a sheaf of $Q(R / x R)$ over $\operatorname{Spec} B(R)$.

Theorem 3.4. Let $R$ be a ring with the Ore condition. Then $Q(R)$ is isomorphic with the ring of sections from $\operatorname{Spec} B(R)$ to the sheaf of $Q(R / x R)$ for $x$ in $\operatorname{Spec} B(R)$.

Proof. Let $F$ be a map from $Q(R)$ to the ring of sections such that $F\left(s^{-1} r\right)=f_{s^{-1} r}$, where $f_{s^{-1} r}(x)=\left(s_{x}\right)^{-1} r_{x}$ for $x$ in Spec $B(R)$; that is, $f_{s^{-1} r}$ is a section induced by $s^{-1} r$. Since the sheaf of $Q(R / x R)$ is a sheaf of $R / x R$-modules over Spec $B(R)$, the map $F$ is a module isomorphism ([9], Theorem 4.5). Hence it suffices to show that $F$ preserves the multiplication. Let $s^{-1} r$ and $s_{1}^{-1} r_{1}$ be in $Q(R)$. We have $F\left(s^{-1} r s_{1}^{-1} r_{1}\right)=$ $f_{s^{-1} r} f_{s_{1}{ }^{-1} r_{1}}$, since $\left(s^{-1} r s_{1}^{-1} r_{1}\right)_{x}=\left(s^{-1} r\right)_{x}\left(s_{1}^{-1} r_{1}\right)_{x}=\left(s_{x}\right)^{-1} r_{x}\left(s_{1}\right)_{x}{ }^{-1}\left(r_{1}\right)_{x}$ by noting that $Q(R / x R) \cong Q(R) / x Q(R)$ by Lemma 3.3. Thus $F\left(s^{-1} r s_{1}{ }^{-1} r_{1}\right)$ $=F\left(s^{-1} r\right) F\left(s_{1}^{-1} r_{1}\right)$.

By [9], the ring $Q(R)$ is isomorphic with the ring of sections of the sheaf induced by $Q(R)$, so we have:

Corollary 3.5. The ring of sections of the sheaf of $Q(R) / y Q(R)$ over $\operatorname{Spec} B(Q(R))$ ( $y$ in $\operatorname{Spec} B(Q(R)))$ is isomorphic with the ring of sections of $Q(R / x R)$ over $\operatorname{Spec} B(R)$ (x in $\operatorname{Spec} B(R))$.

Next are generalizations of the Goldie theorems on quotient rings.

TheOREM 3.6. Let $R$ be a ring with the Ore condition. If $R / x R$ is prime Goldie for each $x$ in Spec $B(R)$, then $Q(R)$ is A-biregular. Conversely, if $Q(R)$ is A-biregular such that $B(Q(R))=B(R)$, then $R / x R$ is prime Goldie for each $x$.

Proof. Let $R / x R$ be prime Goldie for each $x$ in $\operatorname{Spec} B(R)$. Then by the first Goldie theorem ([7], Theorem 4.4) $Q(R / x R)$ is Artinian simple. Hence $Q(R / x R)$ has no central idempotents but $0_{x}$ and $1_{x}$, and so $B(Q(R))=B(R)$. In fact, let $e$ be in $B(Q(R))$. We have $e_{x}=0_{x}$ or $1_{x}$, which are central elements in $R / x R$ for each $x$ in Spec $B(R)$. Hence $e$ is central in $R$; that is, $e$ is in $B(R)$. But then the stalk of $Q(R)$ over Spec $B(Q(R)), Q(R) / y Q(R)$, is isomorphic with $Q(R / y R)$ by Lemma 3.3. Since $Q(R / y R)$ is Artinian simple, $Q(R)$ is $A$-biregular.

Conversely, $Q(R)$ is A-biregular, so $Q(R) / y Q(R)$ is Artinian simple for each $y$ in Spec $B(Q(R))$. By hypothesis, $B(Q(R))=B(R)$, so $\operatorname{Spec} B(Q(R))$ 
$=$ Spec $B(R)$. Hence $Q(R / x R)$ is Artinian simple for each $x$. Then, by Theorem 4.5 in [7], $R / x R$ is prime Goldie for each $x$.

Theorem 3.7. Let $R$ be a ring with the Ore condition. If $R / x R$ is semiprime Goldie for each $x$ in Spec $B(R)$, then $Q(R)$ is weakly A-biregular. Conversely, if $Q(R)$ is weakly A-biregular such that for each $x$ in Spec $B(R), x=\bigcap_{i=1}^{n} y_{i}$ for some $y_{i}$ in Spec $B(Q(R))$ and some positive integer $n$, then $R / x R$ is semiprime Goldie for each $x$.

Proof. Let $R / x R$ be semiprime Goldie for each $x$ in $\operatorname{Spec} B(R)$. Then $Q(R / x R)$ is Artinian semisimple by the second Goldie theorem ([7], Theorem 4.7). For a $y$ in $\operatorname{Spec} B(Q(R))$ such that $x \subset y$, since $Q(R) / y Q(R)$ is a homomorphic image of $Q(R / x R)(\cong Q(R) / x Q(R))$, it is Artinian semisimple. Noting that there exists an $x$ in Spec $B(R)$ contained in a given $y$ in $\operatorname{Spec} B(Q(R))$, we conclude that $Q(R)$ is weakly A-biregular.

Conversely, assume $Q(R)$ is weakly A-biregular. Then by definition, $Q(R) / y Q(R)$ is Artinian semisimple for each $y$ in Spec $B(Q(R))$. By hypothesis, for each $x$ in $\operatorname{Spec} B(R), x=\bigcap_{i=1}^{n} y_{i}$, so it is easy to see that $x Q(R)=\cap y_{i} Q(R)$. Since $y_{i} Q(R)$ and $y_{j} Q(R)$ are comaximal, $Q(R / x R) \cong Q(R) / x Q(R) \cong \oplus \sum_{i=1}^{n} Q(R) / y_{i} Q(R)$ by the Chinese Remainder Theorem. Noting that each summand is Artinian semisimple we conclude that $Q(R / x R)$ is a finite direct sum of Artinian simple rings by Wedderburn's theorem. Thus $R / x R$ is semisimple Goldie for each $x$ by Theorem 4.5 in [7].

Remarks. 1. There exist rings $R$ such that $T(r)$ (Prop. 3.1) is both open and closed for every $r$ in $R$. The following example is due to the referee: Let $X$ be the one point compactification of the integers and $R_{x}=D$ where $D$ is a division ring for all $x$ in $X$. Then the ring $R$ (the ring of sections of $X$ to the sheaf of $R_{x}$ ) with $B(R)$ equal to $X$ and stalks $R_{x}$ has the desired property.

2. The condition that $x=\bigcap_{i=1}^{n} y_{i}$ cannot be dropped from the above theorem. For example, let $R$ be the set of all sequences $\left\{\left(a_{i}\right) \mid a_{i}\right.$ is an integer with $a_{i}=a_{j}$ (modulo $\left.\left.n\right)\right\}$ for a fixed positive integer $n$. Then $R$ is a ring with identity under the component-wise addition and multiplication. We have that the quotient ring of $R, Q(R)=\Pi Q_{i}$, is an infinite direct product of rational fields $Q_{i}$. Since $\operatorname{Spec} B(R)$ contains exactly one point $x, R / x R=R$. Clearly, $Q(R)$ is weakly $A$-biregular and $R / x R$ is not Goldie (in fact, it is $A$-biregular).

3 . It is easy to see the following characterization of the condition that $x=\bigcap_{i=1}^{n} y_{i}$ of Theorem 3.7. Assume that each central idempotent 
of $Q(R / x R)$ is lifted to a central idempotent of $Q(R)$. For each $x$ in Spec $B(R), \quad x=\bigcap_{i=1}^{n} y_{i}$ for some $y_{i}$ in Spec $\left.B(Q)\right)$ if and only if $B(Q(R / x R))$ is a finite set, where $R$ is in Theorem 3.7.

4. As an example of Remark 3, let $Q(R)$ be finitely generated over its center as a ring. Then each central idempotent of $Q(R / x R)$ is lifted to a central idempotent of $Q(R)$.

4. Central idempotents. From Theorem 3.6, we note that no extra central idempotents are produced when $R$ passes to $Q(R)$. But $B(Q(R))$ is larger than $B(R)$ in Theorem 3.7. This will be discussed again in Theorem 4.1, and we shall show that the fact $B(Q(R))=B(R)$ characterizes semiprime Goldie stalks $R / x R$ being prime Goldie. Then a necessary and sufficient condition is found for an element $r$ in $R$ such that $s^{-1} r$ is in $B(R)$ for some non-zero-divisor $s$.

Theorem 4.1. Let $R$ be a ring with the Ore condition with $Q(R)$ finitely generated over its center as a ring. If, for some $x$ in Spec $B(R)$, $R / x R$ is semiprime Goldie but not prime, then $B(Q(R))$ is larger than $B(R)$.

Proof. Let $\left\{a_{1}, \cdots, a_{n}\right\}$ be a set of generators of $Q(R)$ over its center. Since $R / x R$ is semiprime Goldie but not prime for some $x$ in Spec $B(R), Q(R / x R)$ is Artinian semisimple but not simple according to the Goldie second theorem ([7], Theorem 4.5 and Theorem 4.7). Hence there exists at least one central idempotent $E_{x}$ different from $0_{x}$ and $1_{x}$ in $Q(R / x R)$. Since $Q(R / x R) \cong Q(R) / x Q(R)$, we can lift $E_{x}$ to an idempotent $E$ in $Q(R)$ by a basic property proved in [10]. But then $E_{x}\left(a_{i}\right)_{x}=\left(a_{i}\right)_{x} E_{x}$ for each $a_{i}$, and this finite set of equations continues to hold over a basic open set $\Gamma(e)$ for some $e$ in $B(R)$ by a standard property of sheaf theory. Thus $e E a_{i}=e a_{i} E$ for each $i$. This implies that $e E$ is a central idempotent of $Q(R)$. Since $(e E)_{x}\left(=E_{x}\right)$ is different from $0_{x}$ and $1_{x}, e E$ is not in $B(R)$.

TheоReм 4.2. Let $R$ be a ring with the Ore condition such that each central idempotent of $Q(R / x R)$ is lifted to a central idempotent of $Q(R)$. Assume $B(Q(R))=B(R)$. If $R / x R$ is semiprime Goldie for each $x$, then $R / x R$ is prime Goldie.

Proof. Since $R / x R$ is semiprime Goldie, $Q(R / x R)$ is Artinian semisimple. Sine each central idempotent of $Q(R / x R)$ is lifted to a central idempotent of $Q(R)$ and $B(Q(R))=B(R), Q(R / x R)$ has no central idempotents but $0_{x}$ and $1_{x}$ by the proof of Theorem 4.1. Hence $Q(R / x R)$ is Artinian simple. Thus $R / x R$ is prime Goldie. 
When $R$ passes to $Q(R)$, we have already known that $B(Q(R))$ may be larger than $B(R)$. A natural question is what elements $r$ in $R$ are such that $s^{-1} r$ are in $B(R)$ for $s$ in $S$, the set of non-zero-divisors of $R$. Denote the right annihilator of $r$ in $R$ by $A(r)$.

Theorem 4.3. Let $R$ be a ring with the Ore condition. Then, for an element $r$ in $R, A(r)=A(e)$ for some $e$ in $B(R)$ if and only if there exists an $s$ in $S$ such that $s^{-1} r=e$ in $B(R)$.

Proof. The sufficiency is clear. Conversely, since $r=r e+r(1-e)$ $=r e=e r(f \circ \mathrm{r} A(r)=A(e)), r=e r$. Next, from the map of $R$ to $R$ induced by $r$ in $R$ such that $a \rightarrow r a$ for $a$ in $R$, we have $R \cong A(r) \oplus r R$ since $A(r)=A(e)=(1-e) R$ by hypothesis. Thus the decomposition of $R$ implies that $r(=e r)$ is a non-zero-divisor of the ring $e R$. So, $((1-e)+e r)$ is invertible in $Q(R)$, and this implies that $(e r)$ is invertible in $Q(e R)$. But then there exists an $s$ in $S$ such that $s^{-1} r=e$, the identity of $Q(e R)$.

\section{BIBLIOGRAPHY}

1. G. Bergman, Hereditary commutative rings and centres of hereditary rings, Proc. London Math. Soc. 23 (1971), 214-236.

2. W. D. Burgess and W. Stephenson, Pierce sheaves of non-commutative rings, Comm. in Algebra 4 (1), (1976), 51-75.

3. F. DeMeyer, Separable polynomials over a commutative ring, Roc. Mtn. J. Math. 2 (1972), 299-310.

4. A. W. Goldie, The structure of prime rings under ascending chain conditions, Proc. London Math. Soc. 8 (1958), 589-608.

$5 . \longrightarrow$ Semiprime rings with maximum conditions, Proc. London Math. Soc. 10 (1960), 201-220.

6. ㄴ., Rings with maximum conditions, Arch. Math. 13 (1962), 213-221.

7. I. N. Herstein, Topics in ring theory, The University of Chicago Press, 1969.

8. A. Magid, Pierce's representation and separable algebras, Ill. J. Math. 15 (1971), 114-121.

9. R. Pierce, Modules over commutative regular rings, Mem. Amer. Math. Soc. 70 (1967).

10. O. Villamayor and D. Zelinsky, Galois theory for rings with infinitely many idempotents, Nagoya J. Math. 35 (1969), 83-98.

Mathematics Department, Bradley University, Peoria, IL 61625 